\title{
English Setter
}

National Cancer Institute

\section{Source}

National Cancer Institute. English Setter. NCI Thesaurus. Code C53875.

The Eng lish Setter is a slim setter with a speckled coat. The long hair is flat and a bit wavy. The coat comes in white with blue, lemon, orange, or brown speckling. Some dogs are tri-color (blue, white and brown). The moderately long pendant ears have a velvety tip. Height: 23-27 inches (58-69 cm.) Weight: $45-80$ pounds (20-36 kg.) 